\title{
Mixed finite element approximation of eddy current problems
}

\section{Journal Article}

\section{Author(s):}

Rodríguez, Ana Alonso; Hiptmair, Ralf; Valli, Alberto

Publication date:

2004-04

Permanent link:

https://doi.org/10.3929/ethz-b-000158446

Rights / license:

In Copyright - Non-Commercial Use Permitted

Originally published in:

IMA Journal of Numerical Analysis 24(2), https://doi.org/10.1093/imanum/24.2.255 


\title{
Mixed finite element approximation of eddy current problems
}

\author{
Ana Alonso Rodríguez† \\ Dipartimento di Matematica, Università di Milano, via Saldini 50, I-20133 Milano, \\ Italy \\ RALF HIPTMAIR $\ddagger$ \\ Seminar for Applied Mathematics, ETH Zürich, CH-8092 Zürich, Switzerland \\ AND \\ Alberto VALLi§ \\ Dipartimento di Matematica, Università di Trento, I-38050 Povo(Trento), Italy
}

[Received on 1 April 2003; revised on 19 September 2003]

\begin{abstract}
Finite element approximations of eddy current problems that are entirely based on the magnetic field $\mathbf{H}$ are haunted by the need to enforce the algebraic constraint curl $\mathbf{H}=\mathbf{0}$ in non-conducting regions. As an alternative to techniques employing combinatorial Seifert (cutting) surfaces, in order to introduce a scalar magnetic potential we propose mixed multi-field formulations, which enforce the constraint in the variational formulation. In light of the fact that the computation of cutting surfaces is expensive, the mixed finite element approximation is a viable option despite the increased number of unknowns.
\end{abstract}

Keywords: eddy current problems; mixed finite elements; Langrange multipliers.

\section{Introduction}

The governing equations of electromagnetic fields and currents $\mathcal{E}, \mathcal{H}, \mathcal{B}, \mathcal{D}, \mathcal{J}$ are Maxwell's equations completed by constitutive laws in order to model the field-matter interaction. In what follows we shall restrict ourselves to the 'Maxwell model of memoryless linear materials with Ohm’s law’ (see Bossavit, 1998):

$$
\begin{aligned}
-\partial_{t} \mathcal{D}+\operatorname{curl} \mathcal{H} & =\mathcal{J}=\mathcal{J}_{e}+\boldsymbol{\sigma} \mathcal{E}, & & \mathcal{D}=\epsilon \mathcal{E} \\
\partial_{t} \mathcal{B}+\operatorname{curl} \mathcal{E} & =0, & & \mathcal{B}=\boldsymbol{\mu} \mathcal{H} .
\end{aligned}
$$

Here $\boldsymbol{\mu}$ is the magnetic permeability, $\boldsymbol{\epsilon}$ the dielectric tensor, and $\boldsymbol{\sigma}$ stands for conductivity. $\boldsymbol{\mu}$ and $\boldsymbol{\epsilon}$ are assumed to be symmetric and uniformly positive definite $3 \times 3$-matrices, whereas $\sigma$ is supposed to be symmetric and uniformly positive definite inside the conducting region $\Omega^{C}$, but vanishes in the 'air region' $\Omega^{I}$. All the material parameters are functions of the spatial variable $\mathbf{x}$ only. Under these circumstances, if the source current $\mathcal{J}_{e}$ is of the form $\mathcal{J}_{e}(t, \mathbf{x})=\operatorname{Re}\left[\mathbf{J}_{e}(\mathbf{x}) \exp (\mathrm{i} \omega t)\right]$, where $\mathbf{J}_{e}$ is a complex-valued vector field

\footnotetext{
†Email: alonso@mat.unimi.it

‡Email: hiptmair@sam.math.ethz.ch

§mail: valli@science.unitn.it
} 
and $\omega \neq 0$ is a fixed angular frequency, the fields $\mathcal{E}, \mathcal{H}, \mathcal{B}, \mathcal{D}$ also have this harmonic dependence on time. The Maxwell model with Ohm's law then assumes the following strong form:

$$
\begin{aligned}
-\mathrm{i} \omega \mathbf{D}+\operatorname{curl} \mathbf{H} & =\mathbf{J}_{e}+\boldsymbol{\sigma} \mathbf{E}, & & \mathbf{D}=\boldsymbol{\epsilon} \mathbf{E}, \\
\mathrm{i} \omega \mathbf{B}+\operatorname{curl} \mathbf{E} & =\mathbf{0}, & & \mathbf{B}=\boldsymbol{H} .
\end{aligned}
$$

The unknowns now are the complex amplitudes $\mathbf{E}, \mathbf{H}, \mathbf{B}, \mathbf{D}$, independent of time.

In many situations it is possible to consider simpler quasi-static models that still offer a sufficiently accurate description of electromagnetic phenomena. The most popular among these simplified models is the so-called 'eddy current model', which consists in neglecting the term $-\mathrm{i} \omega \mathbf{D}$ in (1.2) (Dirks, 1996; Ammari et al., 2000).

Then compliance with Ampère's law entails

$$
\operatorname{div} \mathbf{J}_{e, I}=0 \quad \text { in } \Omega^{I}, \quad \int_{\Gamma^{j}} \mathbf{J}_{e, I} \cdot \mathbf{n} \mathrm{d} S=0, \quad j=1, \ldots, p_{\Gamma},
$$

where $\Gamma^{j}, j=1, \ldots, p_{\Gamma}$, are the connected components of the boundary of $\Omega^{C}$. The latter is denoted by $\Gamma:=\partial \Omega^{C}$. Here and in the sequel we denote by $\mathbf{v}_{L}$ the restriction of a vector field $\mathbf{v}$ to $\Omega^{L}, L=I, C$.

We introduce an artificial computational domain $\Omega \subset \mathbb{R}^{3}$, which is a box containing the conductors and their immediate neighbourhood, big enough so that one can assume a zero field beyond. As before we write $\Omega^{C}$ for the conductor region and $\Omega^{I}:=\Omega \backslash \overline{\Omega^{C}}$. For the sake of simplicity, we assume that $\Omega^{I}$ is connected.

On $\partial \Omega$, homogeneous boundary conditions for either $\mathbf{H}$ or $\mathbf{E}$ are imposed: throughout we will demand

$$
\mathbf{H} \times \mathbf{n}=\mathbf{0} \text { on } \partial \Omega,
$$

modelling a container made by an infinitely permeable magnetic material (see Bossavit, 1998, p. 232; Silvester \& Ferrari, 1990, p. 408). However, with simple modifications our results also hold for the boundary condition

$$
\boldsymbol{\mu H} \cdot \mathbf{n}=0 \text { on } \partial \Omega .
$$

The boundary condition $\mathbf{H} \times \mathbf{n}=\mathbf{0}$ on $\partial \Omega$ implies another compatibility condition for $\mathbf{J}_{e, I}$, namely

$$
\mathbf{J}_{e, I} \cdot \mathbf{n}=0 \quad \text { on } \partial \Omega \text {. }
$$

Obviously, we cannot expect a solution for $\mathbf{E}$ to be unique, because it can be altered by any gradient supported in $\Omega^{I}$ and will still satisfy the equations. However, imposing the constraints

$$
\begin{gathered}
\operatorname{div}\left(\epsilon \mathbf{E}_{I}\right)=0 \text { in } \Omega^{I}, \quad \epsilon \mathbf{E}_{I} \cdot \mathbf{n}=0 \text { on } \partial \Omega, \\
\int_{\Gamma^{j}} \epsilon \mathbf{E}_{I} \cdot \mathbf{n} \mathrm{d} S=0, j=1, \ldots, p_{\Gamma}-1,
\end{gathered}
$$

(that are implied by (1.2)) will restore uniqueness of the solution for $\mathbf{E}$. 
The complete eddy current model we consider in the sequel is then

$$
\begin{gathered}
\operatorname{curl} \mathbf{H}=\mathbf{J}_{e}+\boldsymbol{\sigma} \mathbf{E}, \quad \mathrm{i} \omega \boldsymbol{\mu} \mathbf{H}+\operatorname{curl} \mathbf{E}=\mathbf{0} \quad \text { in } \Omega, \\
\operatorname{div}\left(\epsilon \mathbf{E}_{I}\right)=0 \quad \text { in } \Omega^{I}, \quad \int_{\Gamma^{j}} \boldsymbol{\epsilon} \mathbf{E}_{I} \cdot \mathbf{n} \mathrm{d} S=0, \quad j=1, \ldots, p_{\Gamma}-1, \\
\boldsymbol{\epsilon} \mathbf{E} \cdot \mathbf{n}=0, \quad \mathbf{H} \times \mathbf{n}=\mathbf{0} \quad \text { on } \partial \Omega .
\end{gathered}
$$

The existence and uniqueness of a solution of problem (1.6) has been proved in Alonso Rodríguez et al. (2003b), assuming that the entries of the matrices $\boldsymbol{\mu}$ and $\boldsymbol{\sigma}$ belong to $L^{\infty}(\Omega)$, those of $\epsilon$ belong to $L^{\infty}\left(\Omega^{I}\right)$, and finally that the source current $\mathbf{J}_{e}$ belongs to $\left(L^{2}(\Omega)\right)^{3}$ and satisfies (1.3) and (1.4).

Dropping the displacement current converts Ampère's law into the purely algebraic constraint curl $\mathbf{H}=\mathbf{J}_{e, I}$ in $\Omega^{I}$. This raises problems not encountered with the full Maxwell equations. This paper will be devoted to how to deal with these problems in the context of a variational formulation based on the magnetic field $\mathbf{H}$. We will focus on approaches that forgo the 'direct option' to incorporate the constraint into the trial space. Instead it is enforced by means of augmented variational equations.

Adding extra equations may seem wasteful and, indeed, it is, because the resulting formulations will, after a finite element Galerkin discretization, feature many additional degrees of freedom. However, this is the price to pay for avoiding the cumbersome 'topological preprocessing', that is the construction of cuts (Gross \& Kotiuga, 2001), that is indispensable in the case of the 'direct option'. Hence, these augmented formulations can become relevant for practical computations. Here we are going to present a couple of possibilities to take into account the seemingly simple constraint curl $\mathbf{H}=\mathbf{J}_{e, I}$ in $\Omega^{I}$. Each variant will come with its own issues of stability and uniqueness.

A brief outline of the paper is as follows: in the next section we introduce notation and function spaces needed for the remainder of the article. Then we review the wellknown H-based variational formulation of the eddy current problem. From these basic equations we derive augmented mixed formulations in Section 4. In Section 5 their finite element Galerkin discretization will be discussed. Finally, in Section 6 we give a priori error estimates.

\section{Basic concepts}

As usual, we denote by $H^{s}(\Omega)$ or $H^{s}(\partial \Omega), s \in \mathbb{R}$, the Sobolev space of order $s$ of real or complex measurable functions defined on $\Omega$ or $\partial \Omega$, respectively. If $\Sigma \subset \partial \Omega$ we denote by $H_{0, \Sigma}^{1}(\Omega)$ the subspace of $H^{1}(\Omega)$ consisting of those functions $\varphi$ satisfying $\varphi_{\mid \Sigma}=0$. As usual $H_{0}^{1}(\Omega):=H_{0, \partial \Omega}^{1}(\Omega)$.

The space $H(\mathbf{c u r l} ; \Omega)$ (respectively, $H(\operatorname{div} ; \Omega)$ ) denotes the set of real or complex vector valued functions $\mathbf{v} \in\left(L^{2}(\Omega)\right)^{3}$ such that curl $\mathbf{v} \in\left(L^{2}(\Omega)\right)^{3}$ (respectively, div $\mathbf{v} \in$ $L^{2}(\Omega)$ ). If $\Sigma \subset \partial \Omega$, by $H_{0, \Sigma}$ (curl; $\Omega$ ) we denote the subspace of $H$ (curl; $\Omega$ ) of those functions v satisfying $(\mathbf{v} \times \mathbf{n})_{\mid \Sigma}=\mathbf{0}$. We set $H_{0}(\mathbf{c u r l} ; \Omega):=H_{0, \partial}(\mathbf{c u r l} ; \Omega)$. $H^{0}(\mathbf{c u r l} ; \Omega)$ denotes the subspace of curl-free functions of $H($ curl; $\Omega)$ and $H_{0, \Sigma}^{0}($ curl; $\Omega)=$ $H_{0, \Sigma}(\mathbf{c u r l} ; \Omega) \cap H^{0}(\mathbf{c u r l} ; \Omega)$. Analogously $H_{0, \Sigma}(\operatorname{div} ; \Omega)$ stands for the subspace of $H(\operatorname{div} ; \Omega)$ containing functions $\mathbf{v}$ satisfying $(\mathbf{v} \cdot \mathbf{n})_{\mid \Sigma}=0$. As above, we set $H_{0}(\operatorname{div} ; \Omega):=$ 
$H_{0, \partial \Omega}(\operatorname{div} ; \Omega)$. Moreover, $H^{0}(\operatorname{div} ; \Omega)$ denotes the subspace of divergence-free functions of $H(\operatorname{div} ; \Omega)$ and $H_{0, \Sigma}^{0}(\operatorname{div} ; \Omega):=H_{0, \Sigma}(\operatorname{div} ; \Omega) \cap H^{0}(\operatorname{div} ; \Omega)$. Finally, $H^{s}(\mathbf{c u r l} ; \Omega)$ denotes the space of vector functions $\mathbf{v} \in\left(H^{s}(\Omega)\right)^{3}$ such that $\operatorname{curl} \mathbf{v} \in\left(H^{s}(\Omega)\right)^{3}$.

Topology enters our considerations through the space of harmonic vector fields

$$
\mathcal{H}:=H_{0, \partial \Omega}^{0}\left(\operatorname{curl} ; \Omega^{I}\right) \cap H_{0, \Gamma}^{0}\left(\operatorname{div} ; \Omega^{I}\right) .
$$

Moreover, for the sake of brevity, we introduce the space of admissible electric fields

$$
W^{I}:=\left\{\mathbf{N}_{I} \in\left(L^{2}\left(\Omega^{I}\right)\right)^{3} \mid \mathbf{N}_{I} \text { satisfies (1.5) }\right\},
$$

and the 'space of unique vector potentials'

$$
Y^{I}:=H_{0, \partial \Omega}\left(\mathbf{c u r l} ; \Omega^{I}\right) \cap H_{0, \Gamma}^{0}\left(\operatorname{div} ; \Omega^{I}\right) \cap \mathcal{H}^{\perp} .
$$

They owe their name to the following result, which will be useful in the sequel. It is essentially contained in Alonso \& Valli (1996) and Fernandes \& Gilardi (1997).

THEOREM 2.1 For each $\mathbf{v}_{I} \in W^{I}$ there is a unique $\mathbf{q}_{I} \in Y^{I}$ such that $\operatorname{curl} \mathbf{q}_{I}=\epsilon \mathbf{v}_{I}$ and

$$
\left\|\mathbf{q}_{I}\right\|_{L^{2}\left(\Omega^{I}\right)} \leqslant C_{1}\left\|\boldsymbol{\epsilon} \mathbf{v}_{I}\right\|_{L^{2}\left(\Omega^{I}\right)} .
$$

\section{The H-based variational formulation}

Basically, two different variational formulations of (1.6) exist, either based on the electric field $\mathbf{E}$ or the magnetic field $\mathbf{H}$ (Bossavit, 1985). They correspond to the primal and dual formulation of a second order elliptic problem. However, the algebraic constraint on curl $\mathbf{H}$ manifests itself in a entirely different way in the two formulations. Therefore we restrict ourselves to the $\mathbf{H}$-based approach.

The generic form of the $\mathbf{H}$-based variational formulation involves the Hilbert space of complex-valued vector functions

$$
V^{0}:=\left\{\mathbf{v} \in H_{0}(\operatorname{curl} ; \Omega) \mid \operatorname{curl}_{I}=\mathbf{0} \text { in } \Omega^{I}\right\},
$$

endowed with the natural norm

$$
\|\mathbf{v}\|_{V^{0}}^{2}:=\int_{\Omega}|\mathbf{v}|^{2}+\int_{\Omega^{C}}\left|\operatorname{curl} \mathbf{v}_{C}\right|^{2}
$$

(recall that $\mathbf{v}_{I}$ and $\mathbf{v}_{C}$ denote the restrictions of $\mathbf{v}$ to $\Omega^{I}$ and $\Omega^{C}$, respectively).

We will also need the affine space

$$
V^{\mathbf{J}_{e, I}}:=\left\{\mathbf{v} \in H_{0}(\operatorname{curl} ; \Omega) \mid \operatorname{curl}_{I}=\mathbf{J}_{e, I} \text { in } \Omega^{I}\right\}=\mathbf{H}^{*}+V^{0},
$$

where $\mathbf{H}^{*}$ is a function in $H_{0}(\mathbf{c u r l} ; \Omega)$ such that $\operatorname{curl} \mathbf{H}_{I}^{*}=\mathbf{J}_{e, I}$ in $\Omega^{I}$. The magnetic field we are looking for belongs to $V^{\mathbf{J}_{e, I}}$. Moreover for each $\mathbf{v} \in V^{0}$

$$
0=\int_{\Omega}(\mathrm{i} \omega \boldsymbol{\mu} \mathbf{H}+\operatorname{curl} \mathbf{E}) \cdot \overline{\mathbf{v}}=i \omega \int_{\Omega} \boldsymbol{\mu H} \cdot \overline{\mathbf{v}}+\int_{\Omega^{C}} \mathbf{E}_{C} \cdot \operatorname{curl} \overline{\mathbf{v}}_{C} .
$$


Using the strong form of Ampère's law in the conductor, namely $\mathbf{E}_{C}=\sigma^{-1}\left(\operatorname{curl} \mathbf{H}_{C}-\right.$ $\mathbf{J}_{e, C}$ ), we arrive at

$$
0=\mathrm{i} \omega \int_{\Omega} \mu \mathbf{H} \cdot \overline{\mathbf{v}}+\int_{\Omega^{C}} \sigma^{-1}\left(\operatorname{curl} \mathbf{H}_{C}-\mathbf{J}_{e, C}\right) \cdot \operatorname{curl} \overline{\mathbf{v}}_{C} .
$$

So, the magnetic field $\mathbf{H}$ solves the following problem:

$$
\left\{\begin{array}{l}
\text { Find } \mathbf{H} \in V^{\mathbf{J}_{e, I}}: \\
\mathrm{i} \omega \int_{\Omega} \mu \mathbf{H} \cdot \overline{\mathbf{v}}+\int_{\Omega^{C}} \sigma^{-1} \operatorname{curl} \mathbf{H}_{C} \cdot \operatorname{curl} \overline{\mathbf{v}}_{C} \\
=\int_{\Omega^{C}} \sigma^{-1} \mathbf{J}_{e, C} \cdot \operatorname{curl} \overline{\mathbf{v}}_{C} \quad \forall \mathbf{v} \in V^{0} .
\end{array}\right.
$$

The existence and uniqueness of a solution of (3.1) follows from the Lax-Milgram lemma, since, under our assumptions on the material coefficients, the bilinear form is trivially coercive on $V^{0}$. Next, we have to recover the electric field in $\Omega$. In $\Omega^{C}$, from Ampère's law we have

$$
\mathbf{E}_{C}=\sigma^{-1}\left(\operatorname{curl} \mathbf{H}_{C}-\mathbf{J}_{e, C}\right),
$$

while in $\Omega^{I}$ there exists a unique $\mathbf{E}_{I} \in H\left(\mathbf{c u r l} ; \Omega^{I}\right)$ such that

$$
\begin{gathered}
\operatorname{curl} \mathbf{E}_{I}=-\mathrm{i} \omega \boldsymbol{\mu} \mathbf{H}_{I}, \quad \operatorname{div}\left(\epsilon \mathbf{E}_{I}\right)=0 \quad \text { in } \Omega^{I}, \\
\epsilon \mathbf{E}_{I} \cdot \mathbf{n}=0 \quad \text { on } \partial \Omega, \quad \int_{\Gamma^{j}} \epsilon \mathbf{E}_{I} \cdot \mathbf{n} \mathrm{d} S=0 \quad j=1, \ldots, p_{\Gamma}-1, \\
\mathbf{E}_{I} \times \mathbf{n}_{I}=-\mathbf{E}_{C} \times \mathbf{n}_{C} \quad \text { on } \Gamma .
\end{gathered}
$$

Here, $\mathbf{n}_{L}$ denotes the unit outward normal vector on $\partial \Omega_{L}, L=I, C$. We refer to Alonso Rodríguez et al. (2003b) for more details.

Then (H, E) with $\mathbf{H}$ solution of (3.1) and $\mathbf{E}$ defined as

$$
\mathbf{E}= \begin{cases}\mathbf{E}_{C} & \text { in } \Omega^{C} \\ \mathbf{E}_{I} & \text { in } \Omega^{I}\end{cases}
$$

is the unique solution of (1.6).

REMARK 3.1 We note that a finite element method based on (3.1) would have to deal with the constrained space $V^{\mathbf{J}_{e, I}}$. The direct way to deal with the constraint in $V^{0}$ makes use of scalar magnetic potentials by representing

$$
V^{0}{ }_{\mid \Omega^{I}}=\operatorname{grad} H_{\partial \Omega}^{1}\left(\Omega^{I}\right) \oplus \mathcal{H}
$$

(see Alonso Rodríguez et al., 2003a; Bermúdez et al., 2002). It would be a perfect solution, unless we had to construct a basis of $\mathcal{H}$ in order to continue with discretization. Such a basis is readily available, once we have 'cuts' at our disposal, i.e. a collection of surfaces in $\Omega^{I}$ that cut any non-bounding cycle (Bossavit, 1998; Tarhasaari \& Kettunen, 2001). Finding these cuts for arbitrary shape of $\Omega^{C}$ seems to be a challenging problem (Gross \& Kotiuga, 2001). 


\section{Mixed formulations}

The main idea is to reformulate (3.1) as a saddle point problem in non-constrained vector spaces by introducing Lagrange multipliers.

Let us define the bilinear form in $H_{0}($ curl; $\Omega)$

$$
a(\mathbf{w}, \mathbf{v}):=\mathrm{i} \omega \int_{\Omega} \boldsymbol{\mu w} \cdot \overline{\mathbf{v}}+\int_{\Omega^{C}} \sigma^{-1} \operatorname{curl} \mathbf{w}_{C} \cdot \operatorname{curl} \overline{\mathbf{v}}_{C} .
$$

We can introduce a Lagrange multiplier $\mathbf{A}_{I} \in\left(L^{2}\left(\Omega^{I}\right)\right)^{3}$ and consider the saddle point problem

$$
\left\{\begin{aligned}
\text { Find }\left(\mathbf{H}, \mathbf{A}_{I}\right) \in H_{0}(\operatorname{curl} ; \Omega) \times\left(L^{2}\left(\Omega^{I}\right)\right)^{3}: & \\
a(\mathbf{H}, \mathbf{v})+\int_{\Omega^{I}} \operatorname{curl} \overline{\mathbf{v}}_{I} \cdot \mathbf{A}_{I} & \\
\quad=\int_{\Omega^{C}} \boldsymbol{\sigma}^{-1} \mathbf{J}_{e, C} \cdot \operatorname{curl} \overline{\mathbf{v}}_{C} & \forall \mathbf{v} \in H_{0}(\operatorname{curl} ; \Omega) \\
\int_{\Omega^{I}} \operatorname{curl~}_{I} \cdot \overline{\mathbf{N}}_{I}=\int_{\Omega^{I}} \mathbf{J}_{e, I} \cdot \overline{\mathbf{N}}_{I} & \forall \mathbf{N}_{I} \in\left(L^{2}\left(\Omega^{I}\right)\right)^{3} .
\end{aligned}\right.
$$

This problem does not have a unique solution as it is possible to add any function of $H_{0, \Gamma}^{0}\left(\mathbf{c u r l} ; \Omega^{I}\right)$ to $\mathbf{A}_{I}$. However, if $\left(\mathbf{H}, \mathbf{A}_{I}\right)$ is a solution of (4.1) then $\mathbf{H}$ is the solution of (3.1), and $\mathbf{A}_{I}$ satisfies curl $\mathbf{A}_{I}=-\mathrm{i} \omega \boldsymbol{\mu} \mathbf{H}_{I}=\operatorname{curl} \mathbf{E}_{I}$ and $\mathbf{A}_{I} \times \mathbf{n}_{I}=$ $\boldsymbol{\sigma}^{-1}\left(\mathbf{J}_{e, C}-\operatorname{curl} \mathbf{H}_{C}\right) \times \mathbf{n}_{C}=-\mathbf{E}_{C} \times \mathbf{n}_{C}=\mathbf{E}_{I} \times \mathbf{n}_{I}$ on $\Gamma$. Thus, in order to restore uniqueness of solution it is natural to look for $\mathbf{A}_{I}$ in the constrained space $W^{I}$ defined in (2.2). Then it is obvious that $\mathbf{A}_{I}=\mathbf{E}_{I}$. From Theorem 2.1 it is easily verified that $W^{I}$ is equal to the range space $\boldsymbol{\epsilon}^{-1}$ curl $H_{0, \partial \Omega}\left(\right.$ curl; $\left.\Omega^{I}\right)$.

Thus, we consider the two-field formulation:

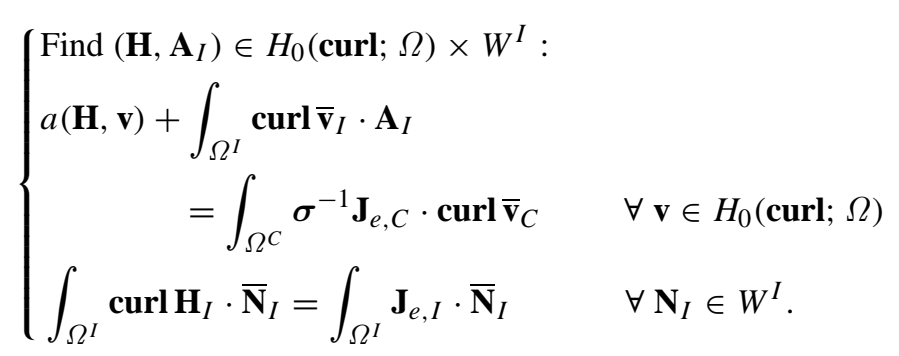

THEOREM 4.1 A unique solution of (4.2) exists.

Proof. We can appeal to Theorem 2.1 and the general theory of variational saddle point problems (Brezzi \& Fortin, 1991).

Again, the space $W^{I}$ involves some constraints. So we introduce another Lagrange multiplier to impose these: we consider the space

$$
H_{*}^{1}\left(\Omega^{I}\right):=\left\{\varphi \in H^{1}\left(\Omega^{I}\right) \mid \varphi_{\mid \Gamma^{j}} \text { is constant } \forall j=1, \ldots, p_{\Gamma}-1, \quad \varphi_{\mid \Gamma^{p}} \Gamma=0\right\},
$$


and it is easily verified by integration by parts that $\mathbf{N}_{I} \in W^{I}$ if and only if $\mathbf{N}_{I} \in\left(L^{2}\left(\Omega^{I}\right)\right)^{3}$ and $\int_{\Omega^{I}} \in \mathbf{N}_{I} \cdot \nabla \bar{\psi}_{I}=0$ for all $\psi_{I} \in H_{*}^{1}\left(\Omega^{I}\right)$. Eventually we confront the following problem:

$$
\left\{\begin{array}{rr}
\text { Find }\left(\mathbf{H}, \mathbf{A}_{I}, \phi_{I}\right) \text { in } H_{0}(\mathbf{c u r l} ; \Omega) \times\left(L^{2}\left(\Omega^{I}\right)\right)^{3} \times H_{*}^{1}\left(\Omega^{I}\right): \\
a(\mathbf{H}, \mathbf{v})+\int_{\Omega^{I}} \operatorname{curl} \overline{\mathbf{v}}_{I} \cdot \mathbf{A}_{I} & \\
=\int_{\Omega^{C}} \sigma^{-1} \mathbf{J}_{e, C} \cdot \operatorname{curl} \overline{\mathbf{v}}_{C} & \forall \mathbf{v} \in H_{0}(\operatorname{curl} ; \Omega) \\
\int_{\Omega^{I}} \operatorname{curl} \mathbf{H}_{I} \cdot \overline{\mathbf{N}}_{I}+\int_{\Omega^{I}} \epsilon \overline{\mathbf{N}}_{I} \cdot \nabla \phi_{I} & \\
=\int_{\Omega^{I}} \mathbf{J}_{e, I} \cdot \overline{\mathbf{N}}_{I} & \forall \mathbf{N}_{I} \in\left(L^{2}\left(\Omega^{I}\right)\right)^{3} \\
\int_{\Omega^{I}} \epsilon \mathbf{A}_{I} \cdot \nabla \bar{\psi}_{I}=0 & \forall \psi_{I} \in H_{*}^{1}\left(\Omega^{I}\right) .
\end{array}\right.
$$

We note that if $\left(\mathbf{H}, \mathbf{A}_{I}, \phi_{I}\right)$ is a solution of (4.3) then $\phi_{I}=0$ (just take $\mathbf{N}_{I}=\nabla \phi_{I}$ in (4.3)), and ( $\left.\mathbf{H}, \mathbf{A}_{I}\right)$ is solution of (4.2).

Introducing the bilinear forms

$$
b(\cdot, \cdot): H_{0, \partial \Omega}\left(\operatorname{curl} ; \Omega^{I}\right) \times\left(L^{2}\left(\Omega^{I}\right)\right)^{3} \rightarrow \mathbb{C}, \quad b\left(\mathbf{v}_{I}, \mathbf{N}_{I}\right):=\int_{\Omega^{I}} \operatorname{curl} \cdot \overline{\mathbf{N}}_{I},
$$

and

$$
c(\cdot, \cdot):\left(L^{2}\left(\Omega^{I}\right)\right)^{3} \times H_{*}^{1}\left(\Omega^{I}\right) \rightarrow \mathbb{C}, \quad c\left(\mathbf{N}_{I}, \psi_{I}\right):=\int_{\Omega^{I}} \epsilon \mathbf{N}_{I} \cdot \nabla \bar{\psi}_{I},
$$

and the linear operators

$$
F(\mathbf{v}):=\int_{\Omega^{C}} \sigma^{-1} \mathbf{J}_{e, C} \cdot \operatorname{curl} \overline{\mathbf{v}}_{C}, \quad \mathbf{v} \in H_{0}(\operatorname{curl} ; \Omega)
$$

and

$$
G\left(\mathbf{N}_{I}\right):=\int_{\Omega^{I}} \mathbf{J}_{e, I} \cdot \overline{\mathbf{N}}_{I}, \quad \mathbf{N}_{I} \in\left(L^{2}\left(\Omega^{I}\right)\right)^{3},
$$

problem (4.3) can be rewritten as

$$
\left\{\begin{array}{lll}
\text { Find }\left(\mathbf{H}, \mathbf{A}_{I}, \phi_{I}\right) \text { in } H_{0}(\mathbf{c u r l} ; \Omega) \times\left(L^{2}\left(\Omega^{I}\right)\right)^{3} \times H_{*}^{1}\left(\Omega^{I}\right): \\
a(\mathbf{H}, \mathbf{v})+\overline{b\left(\mathbf{v}_{I}, \mathbf{A}_{I}\right)}=F(\mathbf{v}) & \forall \mathbf{v} \in H_{0}(\mathbf{c u r l} ; \Omega) \\
b\left(\mathbf{H}_{I}, \mathbf{N}_{I}\right)+\overline{c\left(\mathbf{N}_{I}, \phi_{I}\right)}= & G\left(\mathbf{N}_{I}\right) & \forall \mathbf{N}_{I} \in\left(L^{2}\left(\Omega^{I}\right)\right)^{3} \\
c\left(\mathbf{A}_{I}, \psi_{I}\right) & =0 & \forall \psi_{I} \in H_{*}^{1}\left(\Omega^{I}\right) .
\end{array}\right.
$$

In order to prove that (4.3) has a unique solution, we can use the following result, which is Lemma 4.1 in Chen et al. (2000) extended to complex Hilbert spaces. 
Lemma 4.2 Let $X, Q, M$ be three complex Hilbert spaces and $a: X \times X \rightarrow \mathbb{C}$, $b: X \times Q \rightarrow \mathbb{C}, c: Q \times M \rightarrow \mathbb{C}$ be three continuous bilinear forms, i.e. there exist three positive constants $c_{1}, c_{2}, c_{3}$ such that $|a(\mathbf{v}, \mathbf{w})| \leqslant c_{1}\|\mathbf{v}\|_{X}\|\mathbf{w}\|_{X},|b(\mathbf{v}, \mathbf{N})| \leqslant$ $c_{2}\|\mathbf{v}\|_{X}\|\mathbf{N}\|_{Q},|c(\mathbf{N}, \psi)| \leqslant c_{3}\|\mathbf{N}\|_{Q}\|\psi\|_{M}$ for all $\mathbf{v}, \mathbf{w} \in X, \mathbf{N} \in Q$ and $\psi \in M$. Given $f \in X^{\prime}, g \in Q^{\prime}, l \in M^{\prime}$, let us consider the saddle point problem

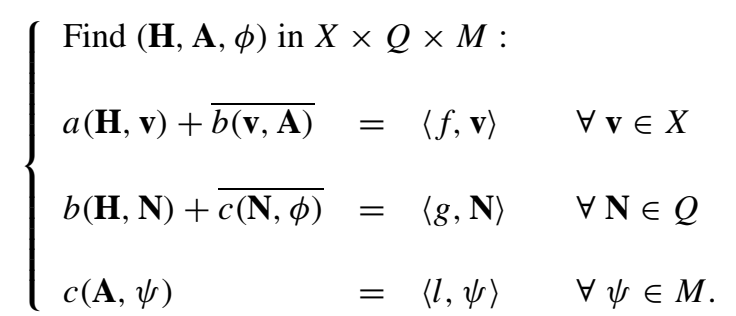

Let $Q^{0} \subset Q$ and $X^{0} \subset X$ be two subspaces as follows:

$$
Q^{0}=\{\mathbf{N} \in Q \mid c(\mathbf{N}, \psi)=0 \quad \forall \psi \in M\}, \quad X^{0}=\left\{\mathbf{v} \in X \mid b(\mathbf{v}, \mathbf{N})=0 \quad \forall \mathbf{N} \in Q^{0}\right\} .
$$

Assume that $a(\cdot, \cdot)$ is $X^{0}$-coercive, i.e.

$$
|a(\mathbf{v}, \mathbf{v})| \geqslant \alpha\|\mathbf{v}\|_{X}^{2} \quad \forall \mathbf{v} \in X^{0},
$$

and that the following inf-sup conditions hold:

$$
\begin{aligned}
& \inf _{\mathbf{N} \in Q^{0}} \sup _{\mathbf{v} \in X} \frac{|b(\mathbf{v}, \mathbf{N})|}{\|\mathbf{v}\|_{X}\|\mathbf{N}\|_{Q}} \geqslant \beta, \\
& \inf _{\psi \in M} \sup _{\mathbf{N} \in Q} \frac{|c(\mathbf{N}, \psi)|}{\|\mathbf{N}\|_{Q}\|\psi\|_{M}} \geqslant \gamma,
\end{aligned}
$$

for some positive constants $\alpha, \beta, \gamma$. Then problem (4.4) has a unique solution.

Now we are in a position to prove the following result.

THEOREM 4.3 Problem (4.3) has a unique solution.

Proof. In order to verify the assumptions of Lemma 4.2, first recall that the spaces $W^{I}$ and $V^{0}$ can also be characterized as

$$
W^{I}=\left\{\mathbf{N}_{I} \in\left(L^{2}\left(\Omega^{I}\right)\right)^{3} \mid \int_{\Omega^{I}} \epsilon \mathbf{N}_{I} \cdot \nabla \bar{\psi}_{I}=0 \quad \forall \psi_{I} \in H_{*}^{1}\left(\Omega^{I}\right)\right\}
$$

and

$$
V^{0}=\left\{\mathbf{v} \in H_{0}(\operatorname{curl} ; \Omega) \mid \int_{\Omega^{I}} \operatorname{curl} \mathbf{v}_{I} \cdot \overline{\mathbf{N}}_{I}=0 \quad \forall \mathbf{N}_{I} \in W^{I}\right\},
$$

(in the latter case, just take $\mathbf{N}_{I}=\epsilon^{-1} \operatorname{curl} \mathbf{v}_{I}$ ).

Since the bilinear form $a(\cdot, \cdot)$ is coercive on the space $V^{0}$, we need only show that the two inf-sup conditions are satisfied, more precisely, that there exist two positive constants $\beta$ and $\gamma$ such that

$$
\sup _{\mathbf{v} \in H_{0}(\mathbf{c u r l} ; \Omega)} \frac{\left|\int_{\Omega^{I}} \operatorname{curl}_{\mathbf{v}} \cdot \overline{\mathbf{N}}_{I}\right|}{\|\mathbf{v}\|_{H(\mathbf{c u r l} ; \Omega)}} \geqslant \beta\left\|\mathbf{N}_{I}\right\|_{L^{2}\left(\Omega^{I}\right)}
$$


for all $\mathbf{N}_{I} \in W^{I}$, and

$$
\sup _{\mathbf{N}_{I} \in\left(L^{2}\left(\Omega^{I}\right)\right)^{3}} \frac{\left|\int_{\Omega^{I}} \epsilon \mathbf{N}_{I} \cdot \nabla \bar{\psi}_{I}\right|}{\left\|\mathbf{N}_{I}\right\|_{L^{2}\left(\Omega^{I}\right)}} \geqslant \gamma\left\|\psi_{I}\right\|_{H^{1}\left(\Omega^{I}\right)}
$$

for all $\psi_{I} \in H_{*}^{1}\left(\Omega^{I}\right)$.

Poincaré's inequality gives us a constant $C_{2}>0$ such that $\left\|\psi_{I}\right\|_{H^{1}\left(\Omega^{I}\right)} \leqslant$ $C_{2}\left\|\nabla \psi_{I}\right\|_{L^{2}\left(\Omega^{I}\right)}$ for all $\psi_{I} \in H_{*}^{1}\left(\Omega^{I}\right)$. Moreover, since $\epsilon$ is assumed to be uniformly positive definite, there exist two positive constants $\epsilon_{*}$ and $\epsilon^{*}$ such that for all $\mathbf{N}_{I} \in$ $\left(L^{2}\left(\Omega^{I}\right)\right)^{3}$

$$
\epsilon_{*}\left\|\mathbf{N}_{I}\right\|_{L^{2}\left(\Omega^{I}\right)}^{2} \leqslant \int_{\Omega^{I}} \epsilon \mathbf{N}_{I} \cdot \mathbf{N}_{I} \leqslant \epsilon^{*}\left\|\mathbf{N}_{I}\right\|_{L^{2}\left(\Omega^{I}\right)}^{2}
$$

Hence, given $\psi_{I} \in H_{*}^{1}\left(\Omega^{I}\right)$ and choosing $\mathbf{N}_{I}=\nabla \psi_{I}$ we have

$\sup _{\mathbf{N}_{I} \in\left(L^{2}\left(\Omega^{I}\right)\right)^{3}} \frac{\left|\int_{\Omega^{I}} \boldsymbol{\epsilon} \mathbf{N}_{I} \cdot \nabla \bar{\psi}_{I}\right|}{\left\|\mathbf{N}_{I}\right\|_{L^{2}\left(\Omega^{I}\right)}} \geqslant \frac{\int_{\Omega^{I}} \epsilon \nabla \psi_{I} \cdot \nabla \bar{\psi}_{I}}{\left\|\nabla \psi_{I}\right\|_{L^{2}\left(\Omega^{I}\right)}} \geqslant \epsilon_{*}\left\|\nabla \psi_{I}\right\|_{L^{2}\left(\Omega^{I}\right)} \geqslant \frac{\epsilon_{*}}{C_{2}}\left\|\psi_{I}\right\|_{H^{1}\left(\Omega^{I}\right)}$.

Concerning (4.8), by Theorem 2.1 for all $\mathbf{N}_{I} \in W^{I}$ there exists $\mathbf{q}_{I} \in Y^{I}$ such that $\mathbf{N}_{I}=\epsilon^{-1}$ curl $\mathbf{q}_{I}$. Let $\mathbf{q} \in H(\operatorname{curl} ; \Omega)$ be a continuous extension of $\mathbf{q}_{I}$ into $\Omega^{C}$; hence, $\|\mathbf{q}\|_{H(\mathbf{c u r l} ; \Omega)} \leqslant C_{3}\left\|\mathbf{q}_{I}\right\|_{H\left(\mathbf{c u r l} ; \Omega^{I}\right)}$. By the stability estimate of Theorem 2.1 we can infer that

$$
\|\mathbf{q}\|_{H(\mathbf{c u r l} ; \Omega)}^{2} \leqslant C_{3}^{2}\left\|\mathbf{q}_{I}\right\|_{H\left(\mathbf{c u r l} ; \Omega^{I}\right)}^{2} \leqslant C_{3}^{2}\left(1+C_{1}^{2}\right)\left\|\operatorname{curl} \mathbf{q}_{I}\right\|_{L^{2}\left(\Omega^{I}\right)}^{2} .
$$

Thus,

$$
\begin{aligned}
& \sup _{\mathbf{v} \in H_{0}(\mathbf{c u r l} \Omega)} \frac{\left|\int_{\Omega^{I}} \operatorname{curl} \mathbf{v}_{I} \cdot \overline{\mathbf{N}}_{I}\right|}{\|\mathbf{v}\|_{H(\operatorname{curl} ; \Omega)} \Omega} \geqslant \frac{\left|\int_{\Omega^{I}} \operatorname{curl} \mathbf{q}_{I} \cdot \overline{\mathbf{N}}_{I}\right|}{\|\mathbf{q}\|_{H(\mathbf{c u r l} ; \Omega)}} \\
& \quad \geqslant \frac{1}{\left(1+C_{1}^{2}\right)^{1 / 2} C_{3}} \frac{\left|\int_{\Omega^{I}} \operatorname{curl} \mathbf{q}_{I} \cdot \overline{\mathbf{N}}_{I}\right|}{\left\|\operatorname{curl} \mathbf{q}_{I}\right\|_{L^{2}\left(\Omega^{I}\right)}}=\frac{1}{\left(1+C_{1}^{2}\right)^{1 / 2} C_{3}} \frac{\int_{\Omega^{I}} \epsilon \mathbf{N}_{I} \cdot \overline{\mathbf{N}}_{I}}{\left\|\epsilon \mathbf{N}_{I}\right\|_{L^{2}\left(\Omega^{I}\right)}} \\
& \quad \geqslant \frac{\epsilon_{*}}{\left(1+C_{1}^{2}\right)^{1 / 2} C_{3} \epsilon^{*}}\left\|\mathbf{N}_{I}\right\|_{L^{2}\left(\Omega^{I}\right)} .
\end{aligned}
$$

\section{Finite element discretization}

We are aiming for Galerkin finite element discretization of both the two-field problem (4.2) and the three-field formulation (4.3). In both cases we want to verify the assumptions of the theory of discrete saddle point problems (Brezzi \& Fortin, 1991, Chapter 2).

We assume that $\Omega, \Omega^{C}, \Omega^{I}$ are Lipschitz polyhedra and consider a family of regular tetrahedral meshes $\left\{\mathcal{T}_{h}\right\}_{h}$ of $\Omega$ such that each element $K \in \mathcal{T}_{h}$ is contained either in $\overline{\Omega^{C}}$ or in $\overline{\Omega^{I}}$. We denote by $\mathcal{T}_{C, h}, \mathcal{T}_{I, h}$ the restriction of $\mathcal{T}_{h}$ to $\Omega^{C}$ and $\Omega^{I}$, respectively. The parameter $h$ will also provide the meshwidth of $\mathcal{T}_{h}$. 
We employ Nédélec's curl-conforming edge elements of the lowest order to approximate the magnetic field: let $V_{h}$ be the finite elements space defined by the complex valued functions

$$
V_{h}:=\left\{\mathbf{v}_{h} \in H(\operatorname{curl} ; \Omega) \mid \mathbf{v}_{h}(\mathbf{x})_{\mid K}=\mathbf{a}_{K}+\mathbf{b}_{K} \times \mathbf{x} \quad \forall K \in \mathcal{T}_{h}\right\},
$$

where $\mathbf{a}_{K}$ and $\mathbf{b}_{K}$ are two constant vectors in $\mathbb{C}^{3}$. It is known that any function $\mathbf{v}_{h} \in V_{h}$ is uniquely determined by the following degrees of freedom (Hiptmair, 2002, Section 3.2):

$$
M_{e}(\mathbf{v})=\left\{\int_{e} \mathbf{v} \cdot \tau \mathrm{d} s \mid e \text { is an edge of } \mathcal{T}_{h}\right\},
$$

where $\tau$ is the unit vector along the edge $e$. These edge moments make sense for any $\mathbf{v} \in\left(H^{s}(\Omega)\right)^{3}$ with curl $\mathbf{v} \in\left(L^{p}(\Omega)\right)^{3}$ with $s>1 / 2$ and $p>2$ (see Amrouche et al., 1998, Lemma 4.7 and Hiptmair, 2002, Lemma 3.13). Moreover, the following interpolation error estimate holds (see Alonso \& Valli, 1999; Chen et al., 2000 and Hiptmair, 2002, Theorem 3.14).

LEMMA 5.1 Denoting by $\pi_{h} \mathbf{w} \in V_{h}$ the interpolant of $\mathbf{w}$, for $1 / 2<s \leqslant 1$, we have

$$
\left\|\pi_{h} \mathbf{w}-\mathbf{w}\right\|_{L^{2}(K)} \leqslant C_{4} h_{K}^{s}\left(\left\|\mathbf{w}_{I}\right\|_{H^{s}(K)}+\left\|\operatorname{curl} \mathbf{w}_{I}\right\|_{H^{s}(K)}\right) \quad \forall \mathbf{w} \in H^{s}(\operatorname{curl} ; K),
$$

where $h_{K}$ is the diameter of $K \in \mathcal{T}_{h}$.

The homogeneoous boundary conditions on $\partial \Omega$ are incorporated by setting degrees of freedom on $\partial \Omega$ to zero. Thus we end up with the spaces

$$
X_{h}:=V_{h} \cap H_{0}(\mathbf{c u r l} ; \Omega) \text { and } X_{h}^{I}:=\left\{\mathbf{v}_{h \mid \Omega^{I}} \mid \mathbf{v}_{h} \in X_{h}\right\} .
$$

For additional information about edge elements the reader is referred to Hiptmair (2002, Chapter 3), Alonso \& Valli (1999), and Girault \& Raviart (1986, Chapter III, Section 5.3).

\subsection{Two-field formulation}

The challenge is the approximations of the constrained space $W^{I}$. However, we can take the cue from the representation in Theorem 2.1 and lift it into the discrete setting. More precisely, we choose

$$
W_{h}^{I}:=\epsilon^{-1} \operatorname{curl} X_{h}^{I}
$$

as trial space for $W^{I}$. Note that this is a conforming discretization in the sense that $W_{h}^{I} \subset$ $W^{I}$. This results in the following discrete two-field problem:

$$
\left\{\begin{array}{l}
\text { Find }\left(\mathbf{H}_{h}, \mathbf{A}_{I, h}\right) \text { in } X_{h} \times W_{h}^{I}: \\
a\left(\mathbf{H}_{h}, \mathbf{v}_{h}\right)+\int_{\Omega_{I}} \operatorname{curl} \overline{\mathbf{v}}_{I, h} \cdot \mathbf{A}_{I, h} \\
=\int_{\Omega_{C}} \sigma^{-1} \mathbf{J}_{e, C} \cdot \operatorname{curl} \overline{\mathbf{v}}_{C, h} \quad \forall \mathbf{v}_{h} \in X_{h}, \\
\int_{\Omega_{I}} \operatorname{curl} \mathbf{H}_{I, h} \cdot \overline{\mathbf{N}}_{I, h}=\int_{\Omega_{I}} \mathbf{J}_{e, I} \cdot \overline{\mathbf{N}}_{I, h} \quad \forall \mathbf{N}_{I, h} \in W_{h}^{I} .
\end{array}\right.
$$


THEOREM 5.2 Problem (5.1) has a unique solution.

Proof. As in the case of the continuous problem, it is straightforward that the bilinear form $a(\cdot, \cdot)$ is continuous in $X_{h}$ and coercive in

$$
X_{h}^{0}:=\left\{\mathbf{v}_{h} \in X_{h} \mid \int_{\Omega_{I}} \operatorname{curl} \mathbf{v}_{I, h} \cdot \overline{\mathbf{N}}_{I, h}=0 \forall \mathbf{N}_{I, h} \in W_{h}^{I}\right\},
$$

since in particular $\boldsymbol{\epsilon}^{-1} \operatorname{curl}_{\mathbf{l}, h} \in W_{h}^{I}$, so that $\mathbf{v}_{h} \in X_{h}^{0}$ implies curl $\mathbf{v}_{h \mid \Omega^{I}}=\mathbf{0}$.

To prove a uniform discrete inf-sup condition we rely on the following lemma. It is a variant of a discrete Poincaré-Friedrichs inequality, see Hiptmair (2002, Theorem 4.7) for a proof.

Lemma 5.3 Let $X_{h}^{I, 0}:=\left\{\mathbf{v}_{I, h} \in X_{h}^{I} \mid \operatorname{curl} \mathbf{v}_{I, h}=\mathbf{0}\right\}$ and

$$
\left(X_{h}^{I, 0}\right)^{\perp}:=\left\{\mathbf{p}_{I, h} \in X_{h}^{I} \mid \int_{\Omega_{I}} \mathbf{p}_{I, h} \cdot \overline{\mathbf{v}}_{I, h}=0 \forall \mathbf{v}_{I, h} \in X_{h}^{I, 0}\right\} .
$$

Then there exists a positive constant $C_{5}$, independent of $h$, such that for all $\mathbf{p}_{I, h} \in\left(X_{h}^{I, 0}\right)^{\perp}$

$$
\left\|\mathbf{p}_{I, h}\right\|_{L^{2}\left(\Omega_{I}\right)} \leqslant C_{5}\left\|\operatorname{curl} \mathbf{p}_{I, h}\right\|_{L^{2}\left(\Omega_{I}\right)} .
$$

By the definition of $W_{h}^{I}$, for each $\mathbf{N}_{I, h} \in W_{h}^{I}$ there exists $\widehat{\mathbf{q}}_{I, h} \in X_{h}^{I}$ such that $\mathbf{N}_{I, h}=$ $\epsilon^{-1}$ curl $\widehat{\mathbf{q}}_{I, h}$. By projecting on $\left(X_{h}^{I, 0}\right)^{\perp}$, we find a unique $\mathbf{q}_{I, h} \in\left(X_{h}^{I, 0}\right)^{\perp}$ with the same property. Let $\mathbf{q}_{h}$ be some uniform discrete extension of $\mathbf{q}_{I, h}$ to $\Omega^{C}$ (the existence of such an extension has been proved in Alonso \& Valli, 1999). Then $\mathbf{q}_{h} \in X_{h}$ and

$$
\left\|\mathbf{q}_{h}\right\|_{H(\mathbf{c u r l} ; \Omega)} \leqslant C_{6}\left\|\mathbf{q}_{I, h}\right\|_{H\left(\mathbf{c u r l} ; \Omega_{I}\right)} \leqslant C_{6}\left(1+C_{5}^{2}\right)^{1 / 2}\left\|\operatorname{curl} \mathbf{q}_{I, h}\right\|_{L^{2}\left(\Omega_{I}\right)} .
$$

Since the constant $C_{6}\left(1+C_{5}^{2}\right)^{1 / 2}$ is independent of $h, \mathbf{q}_{h}$ is a suitable candidate in the discrete inf-sup condition:

$$
\sup _{\mathbf{v}_{h} \in X_{h}} \frac{\left|\int_{\Omega_{I}} \operatorname{curl}_{I, h} \cdot \overline{\mathbf{N}}_{I, h}\right|}{\left\|\mathbf{v}_{h}\right\|_{H_{0}(\mathbf{c u r l} ; \Omega)}} \geqslant \frac{\left|\int_{\Omega_{I}} \operatorname{curl} \mathbf{q}_{I, h} \cdot \overline{\mathbf{N}}_{I, h}\right|}{\left\|\mathbf{q}_{h}\right\|_{H_{0}(\mathbf{c u r l} ; \Omega)}} \geqslant \frac{\epsilon_{*}}{C_{6}\left(1+C_{5}^{2}\right)^{1 / 2} \epsilon^{*}}\left\|\mathbf{N}_{I, h}\right\|_{L^{2}\left(\Omega_{I}\right)} .
$$

All assumptions of Brezzi \& Fortin (1991, Chapter II, Theorem 1.1) are satisfied.

REMARK 5.1 The only way to implement the space $W_{h}^{I}$ is to rely on its very definition, that is we obtain its elements as $\epsilon^{-1}$ curl of edge element functions. Yet, these will no longer be unique. The bottom line is that in a practical implementation of the two-field method we will face a singular system of linear equations. As its kernel is well separated, conjugate gradient type iterative solvers will perform well.

\subsection{Three-field formulation}

Apart from $\mathbf{H}$ we have to approximate $\mathbf{A}_{I}$ (namely, $\mathbf{E}_{I}$ ) and $\phi_{I}$ in (4.3). Let $P_{k}$ denote the standard space of complex polynomials of total degree less than or equal to $k$ with respect 
to the real variable $\mathbf{x}$. To discretize $\mathbf{A}_{I} \in L^{2}\left(\Omega^{I}\right)$ we choose piecewise constant vector functions in the space

$$
Q_{h}^{I}:=\left\{\mathbf{N}_{I, h} \in\left(L^{2}\left(\Omega^{I}\right)\right)^{3} \mid \mathbf{N}_{I, h \mid K} \in\left(P_{0}\right)^{3} \forall K \in \mathcal{T}_{I, h}\right\} .
$$

In order to approximate the Lagrangian multiplier $\phi_{I} \in H_{*}^{1}\left(\Omega^{I}\right)$ it would be natural to rely on piecewise linear Lagrangian finite elements. However, it turns out that this space is too small to guarantee uniform stability of the discretized mixed formulation. We have to switch to a larger space for the approximation of the Lagrangian multiplier; it will be the non-conforming Crouzeix-Raviart elements, defined as follows:

$$
U_{h}^{I}:=\left\{\psi_{I, h} \in L^{2}\left(\Omega^{I}\right) \mid \psi_{I, h \mid K} \in P_{1} \forall K \in \mathcal{T}_{I, h} \text { and } \psi_{I, h}\right. \text { is continuous at the }
$$
centroid of any face $f$ common to two elements in $\left.\mathcal{T}_{h}\right\}$.

Then the discrete $\phi_{I}$ will belong to

$$
\begin{aligned}
M_{h}^{I} & :=\left\{\psi_{I, h} \in U_{h}^{I} \mid \psi_{I, h}(\mathbf{p}) \text { is equal for all centroids } \mathbf{p} \text { of faces of } \Gamma^{j},\right. \\
j & \left.=1, \ldots, p_{\Gamma}-1, \text { and } \psi_{I, h}(\mathbf{p})=0 \text { for all centroids } \mathbf{p} \text { of faces of } \Gamma^{p}\right\} .
\end{aligned}
$$

Note that, since functions in $U_{h}^{I}$ are no longer continuous, they are no longer in $H^{1}\left(\Omega^{I}\right)$. Therefore we must define a modified bilinear form $c_{h}:\left(L^{2}\left(\Omega^{I}\right)\right)^{3} \times\left(H_{*}^{1}\left(\Omega^{I}\right)+M_{h}^{I}\right) \rightarrow \mathbb{C}$ and a norm on $H_{*}^{1}\left(\Omega^{I}\right)+M_{h}^{I}$. For each $\psi_{I} \in H_{*}^{1}\left(\Omega^{I}\right)+M_{h}^{I}$ we denote $\widetilde{\nabla} \psi_{I}$ the function in $\left(L^{2}\left(\Omega^{I}\right)\right)^{3}$ defined as

$$
\left(\widetilde{\nabla} \psi_{I}\right)_{\mid K}:=\nabla\left(\psi_{I \mid K}\right) \quad \forall K \in \mathcal{T}_{I, h}
$$

Note that if $\psi_{I} \in H_{*}^{1}\left(\Omega^{I}\right)$, then $\widetilde{\nabla} \psi_{I}=\nabla \psi_{I}$. Similarly, we define the bilinear form

$$
\begin{aligned}
c_{h}\left(\mathbf{N}_{I}, \psi_{I}\right) & :=\sum_{K} \int_{K} \epsilon \mathbf{N}_{I} \cdot \nabla \psi_{I} \\
& =\int_{\Omega^{I}} \epsilon \mathbf{N}_{I} \cdot \widetilde{\nabla} \psi_{I} \quad \forall \mathbf{N}_{I} \in\left(L^{2}\left(\Omega^{I}\right)\right)^{3}, \psi_{I} \in H_{*}^{1}\left(\Omega^{I}\right)+M_{h}^{I}
\end{aligned}
$$

and the norm in $H_{*}^{1}\left(\Omega^{I}\right)+M_{h}^{I}$

$$
\left\|\psi_{I}\right\|_{h}^{2}:=\sum_{K} \int_{K}\left|\nabla \psi_{I}\right|^{2}=\left\|\widetilde{\nabla} \psi_{I}\right\|_{L^{2}\left(\Omega^{I}\right)}^{2} .
$$

Then, the finite element approximation of (4.3) can be formulated as follows:

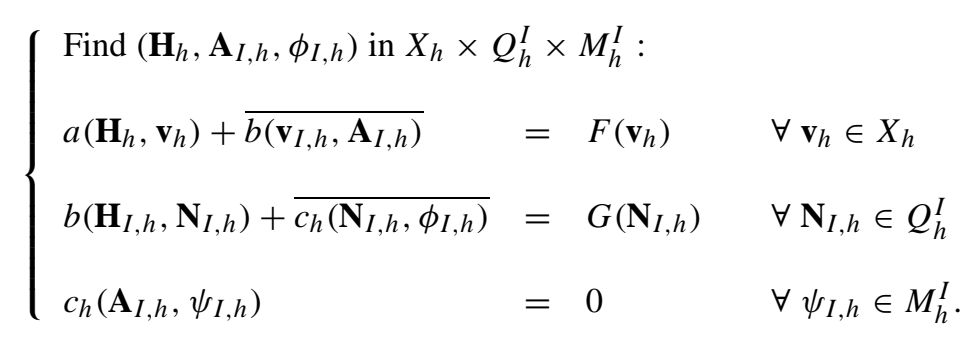

To show that this problem has a unique solution we need the following lemma, (see Monk, 1991). 
LEMMA 5.4 We have the $L^{2}\left(\Omega^{I}\right)$-orthogonal decomposition $Q_{h}^{I}=\operatorname{curl} X_{h}^{I} \oplus \widetilde{\nabla} M_{h}^{I}$.

Proof. The proof has two parts. In the first part we show that for all $\mathbf{v}_{I, h} \in X_{h}^{I}$ and $\psi_{I, h} \in$ $M_{h}^{I}$ we have the orthogonality $\int_{\Omega^{I}} \operatorname{curl}_{I, h} \cdot \widetilde{\nabla} \psi_{I, h}=0$. In the second part we establish that $\operatorname{dim}\left(Q_{h}^{I}\right)=\operatorname{dim}\left(\operatorname{curl} X_{h}^{I}\right)+\operatorname{dim}\left(\widetilde{\nabla} M_{h}^{I}\right)$.

For any $\mathbf{v}_{I, h} \in X_{h}^{I}$ and $\psi_{I, h} \in M_{h}^{I}$ integration by parts yields

$$
\begin{aligned}
\int_{\Omega^{I}} \operatorname{curl}_{I, h} \cdot \tilde{\nabla} \psi_{I, h}= & \sum_{K} \int_{K} \operatorname{curl}_{I, h} \cdot \nabla \psi_{I, h}=\sum_{K} \int_{\partial K} \operatorname{curl}_{I, h} \cdot \mathbf{n} \psi_{I, h} \\
= & \sum_{f \in \mathcal{F}_{\text {int }}} \int_{f} \operatorname{curl}_{I, h} \cdot \mathbf{n}\left[\psi_{I, h}\right]_{f}+\sum_{f \in \mathcal{F}_{\partial \Omega}} \int_{f} \operatorname{curl}_{I, h} \cdot \mathbf{n} \psi_{I, h} \\
& +\sum_{j=1}^{p_{\Gamma}} \sum_{f \in \mathcal{F}_{\Gamma^{j}}} \int_{f} \operatorname{curl}_{I, h} \cdot \mathbf{n} \psi_{I, h},
\end{aligned}
$$

where $\mathcal{F}_{\text {int }}$ is the set of internal faces of the triangulation $\mathcal{T}_{I, h}, \mathcal{F}_{\partial \Omega}$ and $\mathcal{F}_{\Gamma^{j}}$ denote the set of faces of $\mathcal{T}_{I, h}$ on $\partial \Omega$ and $\Gamma^{j}$, respectively, and $\left[\psi_{I, h}\right]_{f}$ denotes the jump of $\psi_{I, h}$ across the face $f$. Note that, for all $f \in \mathcal{F}_{\text {int }},\left(\operatorname{curl}_{\mathbf{I}, h} \cdot \mathbf{n}\right)_{\mid f}$ is constant and $\int_{f}\left[\psi_{I, h}\right]_{f}=$ 0 since $\left[\psi_{I, h}\right]_{f}$ is a linear function and it is equal zero in the centroid of $f$. Moreover $\left(\operatorname{curl}_{I, h} \cdot \mathbf{n}\right)_{\mid f}=0$ for all $f \in \mathcal{F}_{\partial \Omega}$, and, using that for all faces $f \in \mathcal{F}_{\Gamma^{j}}$ one has $\int_{f} \psi_{I, h}=\psi_{j}$ meas $(f)$, for all $j=1, \ldots, p_{\Gamma}$, we finally find

$$
\sum_{f \in \mathcal{F}_{\Gamma^{j}}} \int_{f} \operatorname{curl}_{\mathbf{l}} \mathbf{v}_{I, h} \cdot \mathbf{n} \psi_{I, h}=\sum_{f \in \mathcal{F}_{\Gamma^{j}}}\left(\operatorname{curl}_{I, h} \cdot \mathbf{n}\right)_{\mid f} \psi_{j} \operatorname{meas}(f)=\psi_{j} \int_{\Gamma^{j}} \operatorname{curl}_{I, h} \cdot \mathbf{n}=0,
$$

hence

$$
\int_{\Omega^{I}} \operatorname{curl}_{I, h} \cdot \widetilde{\nabla} \psi_{I, h}=0
$$

Let us introduce the Raviart-Thomas finite element space (Brezzi \& Fortin, 1991, Chapter III)

$$
R T_{h}:=\left\{\mathbf{v}_{h} \in H\left(\operatorname{div} ; \Omega^{I}\right) \mid \mathbf{v}_{h}(\mathbf{x})_{\mid K}=\mathbf{a}_{K}+b_{K} \mathbf{x} \quad \forall K \in \mathcal{T}_{I, h}\right\},
$$

where $\mathbf{a}_{K}$ is a constant complex vector and $b_{K}$ is a complex number, and the subspaces

$$
R T_{0, \partial \Omega}:=R T_{h} \cap H_{0, \partial \Omega}\left(\operatorname{div}, \Omega^{I}\right), \quad R T_{0, \partial \Omega}^{0}:=R T_{h} \cap H_{0, \partial \Omega}^{0}\left(\operatorname{div}, \Omega^{I}\right) .
$$

By arguments from discrete cohomology, it can be proved (see Bossavit, 1998) that, as vector spaces on $\mathbb{C}$,

$$
\operatorname{dim}\left(\operatorname{curl} X_{h}^{I}\right)=\operatorname{dim}\left(R T_{0, \partial \Omega}^{0}\left(\Omega^{I}\right)\right)-\left(p_{\Gamma}-1\right) .
$$

Let us denote by $\# K$ the number of tetrahedra of $\mathcal{T}_{I, h}$, by $\# \mathcal{F}$ the total number of faces of $\mathcal{T}_{I, h}$ and by $\# \mathcal{F}_{\partial \Omega}, \# \mathcal{F}_{\Gamma}$, the number of faces of $\mathcal{T}_{I, h}$ on $\partial \Omega$ and by $\Gamma$ respectively. It is not difficult to prove that

$$
\begin{aligned}
& \operatorname{dim}\left(R T_{0, \partial \Omega}^{0}\right)=\operatorname{dim}\left(R T_{0, \partial \Omega}\left(\Omega^{I}\right)\right)-\operatorname{dim}\left(\operatorname{div}\left(R T_{0, \partial \Omega}\left(\Omega^{I}\right)\right)\right)=\left(\# \mathcal{F}-\# \mathcal{F}_{\partial \Omega}\right)-\# K, \\
& \operatorname{dim}\left(M_{h}^{I}\right)=\left(\# \mathcal{F}-\# \mathcal{F}_{\Gamma}\right)+\left(p_{\Gamma}-1\right)=\operatorname{dim}\left(\widetilde{\nabla}\left(M_{h}^{I}\right)\right), \\
& \operatorname{dim}\left(Q_{h}^{I}\right)=3 \# K
\end{aligned}
$$


Since $4 \# K=2 \# \mathcal{F}-\left(\# \mathcal{F}_{\partial \Omega}+\# \mathcal{F}_{\Gamma}\right)$ then

$$
\begin{aligned}
& \operatorname{dim}\left(\operatorname{curl} X_{h}^{I}\right)+\operatorname{dim}\left(\widetilde{\nabla} M_{h}^{I}\right) \\
& \quad=\left[\left(\# \mathcal{F}-\# \mathcal{F}_{\partial \Omega}\right)-\# K-\left(p_{\Gamma}-1\right)\right]+\left[\left(\# \mathcal{F}-\# \mathcal{F}_{\Gamma}\right)+\left(p_{\Gamma}-1\right)\right] \\
& \quad=2 \# \mathcal{F}-\left(\# \mathcal{F}_{\partial \Omega}+\# \mathcal{F}_{\Gamma}\right)-\# K=4 \# K-\# K=\operatorname{dim}\left(Q_{h}^{I}\right)
\end{aligned}
$$

Since, trivially, curl $X_{h}^{I} \subset Q_{h}^{I}$ and $\widetilde{\nabla} M_{h}^{I} \subset Q_{h}^{I}$, the proof is finished.

Using Lemma 5.3, we can now prove the main result of this section.

THEOREM 5.5 Given a triangulation $\mathcal{T}_{h}$ of $\Omega$, assume that the entries of the matrix $\epsilon$ are piecewise constants in $\Omega^{I}$. Then problem (5.4) has a unique solution.

Proof. Conditions (4.5) and (4.6) follow as in the proof of Theorem 5.2, provided that we show that the space

$$
\left\{\mathbf{v}_{h} \in X_{h} \mid b\left(\mathbf{v}_{I, h}, \mathbf{N}_{I, h}\right)=0 \quad \forall \mathbf{N}_{I, h} \in Q_{h}^{I, 0}\right\}
$$

where $Q_{h}^{I, 0} \subset Q_{h}^{I}$ is defined as follows:

$$
Q_{h}^{I, 0}:=\left\{\mathbf{N}_{I, h} \in Q_{h}^{I} \mid c_{h}\left(\mathbf{N}_{I, h}, \psi_{I, h}\right)=0 \quad \forall \psi_{I, h} \in M_{h}^{I}\right\},
$$

coincides with the space $X_{h}^{0}$ defined in (5.2). In fact, it is enough to prove that $Q_{h}^{I, 0}=W_{h}^{I}$. Since the entries of the matrix $\epsilon$ are piecewise constants, for each $\mathbf{N}_{I, h} \in Q_{h}^{I, 0}$ we have $\epsilon \mathbf{N}_{I, h} \in Q_{h}^{I}$. Therefore, using Lemma 5.4, we obtain that $\epsilon \mathbf{N}_{I, h} \in \operatorname{curl} X_{h}^{I}$, hence $Q_{h}^{I, 0} \subset$ $\epsilon^{-1}$ curl $X_{h}^{I}$. The converse is straightforward, proceeding as in the proof of Lemma 5.4.

Concerning the inf-sup condition (4.7) note that for all $\psi_{I, h} \in M_{h}^{I}$ one has $\widetilde{\nabla} \psi_{I, h} \in$ $Q_{h}^{I}$, hence from the definition of the norm $\|\cdot\|_{h}$

$$
\sup _{\mathbf{N}_{I, h} \in Q_{h}^{I}} \frac{\left|c_{h}\left(\mathbf{N}_{I, h}, \psi_{I, h}\right)\right|}{\left\|\mathbf{N}_{I, h}\right\|_{L^{2}\left(\Omega^{I}\right)}\left\|\psi_{I, h}\right\|_{h}} \geqslant \frac{\left|c_{h}\left(\widetilde{\nabla} \psi_{I, h}, \psi_{I, h}\right)\right|}{\left\|\widetilde{\nabla} \psi_{I, h}\right\|_{L^{2}\left(\Omega^{I}\right)}\left\|\psi_{I, h}\right\|_{h}}=\frac{\int_{\Omega^{I}} \boldsymbol{\epsilon} \widetilde{\nabla} \psi_{I, h} \cdot \widetilde{\nabla} \psi_{I, h}}{\left\|\widetilde{\nabla} \psi_{I, h}\right\|_{L^{2}\left(\Omega^{I}\right)}^{2}} \geqslant \epsilon_{*} .
$$

REMARK 5.2 Note that $\mathbf{J}_{e, I}=\operatorname{curl} \mathbf{K}_{e, I}$ for some $\mathbf{K}_{e, I} \in H_{0, \partial \Omega}\left(\operatorname{curl} ; \Omega^{I}\right)$. If $\pi_{h} \mathbf{K}_{e, I}$ is well defined, we can define $G_{h}\left(\mathbf{N}_{I}\right):=\int_{\Omega^{I}} \operatorname{curl}\left(\pi_{h} \mathbf{K}_{e, I}\right) \cdot \mathbf{N}_{I}$. If in problem (5.4) we replace $G$ with $G_{h}$, it is easily showed that the new $\phi_{I, h}$ is equal to zero.

\section{Error estimates}

Given the discrete inf-sup conditions established in Section 5.1, the quasi-optimality of the discrete solution of the two-field problem is standard (Brezzi \& Fortin, 1991, Chapter 2). Here, we are only concerned with the discrete three-field problem (5.4). 
We denote by $c_{1}$ and $c_{2}$ the continuity constants of the bilinear forms $a(\cdot, \cdot)$ and $b(\cdot, \cdot)$ respectively, by $\alpha$ the coercivity constant of $a(\cdot, \cdot)$ in $V^{0}$ and by $\beta$ and $\gamma$ two positive constants, independent of $h$, such that

$$
\inf _{\mathbf{N}_{I, h} \in Q_{h}^{I, 0}} \sup _{\mathbf{v}_{h} \in X_{h}} \frac{\left|b\left(\mathbf{v}_{I, h}, \mathbf{N}_{I, h}\right)\right|}{\left\|\mathbf{v}_{h}\right\|_{H(\operatorname{curl}, \Omega)}\left\|\mathbf{N}_{I, h}\right\|_{L^{2}\left(\Omega^{I}\right)}} \geqslant \beta,
$$

and

$$
\inf _{\psi_{I, h} \in M_{h}^{I} \mathbf{N}_{I, h} \in Q_{h}} \frac{\left|c_{h}\left(\mathbf{N}_{I, h}, \psi_{I, h}\right)\right|}{\left\|\phi_{I, h}\right\|_{h}\left\|\mathbf{N}_{I, h}\right\|_{L^{2}\left(\Omega^{I}\right)}} \geqslant \gamma
$$

From (5.3) and (5.5) we can take $\beta=\frac{\epsilon_{*}}{C_{6}\left(1+C_{5}^{2}\right)^{1 / 2} \epsilon^{*}}$ and $\gamma=\epsilon_{*}$.

THEOREM 6.1 Let $\left(\mathbf{H}, \mathbf{A}_{I}, \phi_{I}\right) \in H_{0}(\mathbf{c u r l} ; \Omega) \times\left(L^{2}\left(\Omega^{I}\right)\right)^{3} \times H_{*}^{1}\left(\Omega^{I}\right)$ be the solution of problem (4.3) and $\left(\mathbf{H}_{h}, \mathbf{A}_{I, h}, \phi_{I, h}\right) \in X_{h} \times Q_{h}^{I} \times M_{h}^{I}$ the solution of problem (5.4). Then the following error estimates hold:

$$
\begin{gathered}
\left\|\mathbf{H}-\mathbf{H}_{h}\right\|_{H(\text { curl } ; \Omega)} \leqslant\left(1+\frac{c_{1}}{\alpha}\right)\left(1+\frac{c_{2}}{\beta}\right) \inf _{\mathbf{v}_{h} \in X_{h}}\left\|\mathbf{H}-\mathbf{v}_{h}\right\|_{H(\text { curl } ; \Omega)}, \\
\left\|\mathbf{A}_{I}-\mathbf{A}_{I, h}\right\|_{L^{2}\left(\Omega^{I}\right)} \leqslant\left(1+\frac{c_{2}}{\beta}\right) \inf _{\mathbf{N}_{I, h} \in Q_{h}^{I, 0}}\left\|\mathbf{A}_{I}-\mathbf{N}_{I, h}\right\|_{L^{2}\left(\Omega^{I}\right)} \\
+\frac{c_{1}}{\beta}\left\|\mathbf{H}-\mathbf{H}_{h}\right\|_{H(\text { curl } ; \Omega)}, \\
\left\|\phi_{I}-\phi_{I, h}\right\|_{h}=\left\|\phi_{I, h}\right\|_{h} \leqslant \frac{c_{2}}{\gamma}\left\|\mathbf{H}-\mathbf{H}_{h}\right\|_{H(\mathbf{c u r l} ; \Omega)} .
\end{gathered}
$$

Proof. The proof follows the lines of the proofs in Brezzi \& Fortin (1991, Chapter 2). For all $\mathbf{v}_{h}^{*}, \mathbf{v}_{h} \in X_{h}$ and $\mathbf{N}_{I, h} \in Q_{h}^{I}$

$$
\begin{gathered}
a\left(\mathbf{H}_{h}-\mathbf{v}_{h}^{*}, \mathbf{v}_{h}\right)+\overline{b\left(\mathbf{v}_{I, h}, \mathbf{A}_{I, h}-\mathbf{N}_{I, h}\right)}=F\left(\mathbf{v}_{h}\right)-a\left(\mathbf{v}_{h}^{*}, \mathbf{v}_{h}\right)-\overline{b\left(\mathbf{v}_{I, h}, \mathbf{N}_{I, h}\right)} \\
=a\left(\mathbf{H}-\mathbf{v}_{h}^{*}, \mathbf{v}_{h}\right)+\overline{b\left(\mathbf{v}_{I, h}, \mathbf{A}_{I}-\mathbf{N}_{I, h}\right)} .
\end{gathered}
$$

Note that if $\mathbf{v}_{h} \in X_{h}^{0}$ then $\operatorname{curl} \mathbf{v}_{I, h}=\mathbf{0}$ in $\Omega^{I}$, therefore $a\left(\mathbf{H}_{h}-\mathbf{v}_{h}^{*}, \mathbf{v}_{h}\right)=a\left(\mathbf{H}-\mathbf{v}_{h}^{*}, \mathbf{v}_{h}\right)$. If we take $\mathbf{v}_{h}^{*} \in X_{h}^{G}:=\left\{\mathbf{v}_{h} \in X_{h} \mid b\left(\mathbf{v}_{I, h}, \mathbf{N}_{I, h}\right)=G\left(\mathbf{N}_{I, h}\right) \forall \mathbf{N}_{I, h} \in Q_{h}^{I, 0}\right\}$, then $\mathbf{H}_{h}-\mathbf{v}_{h}^{*} \in X_{h}^{0}$ and we find

$$
a\left(\mathbf{H}_{h}-\mathbf{v}_{h}^{*}, \mathbf{H}_{h}-\mathbf{v}_{h}^{*}\right)=a\left(\mathbf{H}-\mathbf{v}_{h}^{*}, \mathbf{H}_{h}-\mathbf{v}_{h}^{*}\right) .
$$

Since $X_{h}^{0} \subset V^{0}$, from coerciveness we conclude

$$
\begin{aligned}
\left\|\mathbf{H}-\mathbf{H}_{h}\right\|_{H(\mathbf{c u r l} ; \Omega)} & \leqslant\left\|\mathbf{H}-\mathbf{v}_{h}^{*}\right\|_{H(\mathbf{c u r l} ; \Omega)}+\left\|\mathbf{H}_{h}-\mathbf{v}_{h}^{*}\right\|_{H(\operatorname{curl} ; \Omega)} \\
& \leqslant\left(1+\frac{c_{1}}{\alpha}\right)\left\|\mathbf{H}-\mathbf{v}_{h}^{*}\right\|_{H(\mathbf{c u r l} ; \Omega)} \quad \forall \mathbf{v}_{h}^{*} \in X_{h}^{G} .
\end{aligned}
$$

Moreover, from the inf-sup condition (6.1), for all $\mathbf{v}_{h} \in X_{h}$ there exists a unique $\mathbf{z}_{h} \in$ $\left(X_{h}^{0}\right)^{\perp}$ such that $b\left(\mathbf{z}_{I, h}, \mathbf{N}_{I, h}\right)=b\left(\mathbf{H}_{I}-\mathbf{v}_{I, h}, \mathbf{N}_{I, h}\right)$ for all $\mathbf{N}_{I, h} \in Q_{h}^{I, 0}$ and

$$
\left\|\mathbf{z}_{h}\right\|_{H(\mathbf{c u r l} ; \Omega)} \leqslant \frac{c_{2}}{\beta}\left\|\mathbf{H}-\mathbf{v}_{h}\right\|_{H(\mathbf{c u r l} ; \Omega)} .
$$


Setting $\mathbf{v}_{h}^{*}:=\mathbf{z}_{h}+\mathbf{v}_{h}$, for all $\mathbf{N}_{I, h} \in Q_{h}^{I, 0}$ we have

$$
b\left(\mathbf{v}_{I, h}^{*}, \mathbf{N}_{I, h}\right)=b\left(\mathbf{H}_{I}, \mathbf{N}_{I, h}\right)=b\left(\mathbf{H}_{I}, \mathbf{N}_{I, h}\right)+\overline{c\left(\mathbf{N}_{I, h}, \phi_{I}\right)}=G\left(\mathbf{N}_{I, h}\right),
$$

hence $\mathbf{v}_{h}^{*} \in X_{h}^{G}$. Furthermore,

$\left\|\mathbf{H}-\mathbf{v}_{h}^{*}\right\|_{H(\mathbf{c u r l} ; \Omega)} \leqslant\left\|\mathbf{H}-\mathbf{v}_{h}\right\|_{H(\mathbf{c u r l} ; \Omega)}+\left\|\mathbf{z}_{h}\right\|_{H(\text { curl } ; \Omega)} \leqslant\left(1+\frac{c_{2}}{\beta}\right)\left\|\mathbf{H}-\mathbf{v}_{h}\right\|_{H(\text { curl } ; \Omega)}$, and (6.3) follows from (6.6).

To obtain (6.4) we use the inf-sup condition (6.1). For each $\mathbf{N}_{I, h} \in Q_{h}^{I, 0}$ we find

$$
\left\|\mathbf{A}_{I, h}-\mathbf{N}_{I, h}\right\|_{L^{2}\left(\Omega^{I}\right)} \leqslant \frac{1}{\beta} \sup _{\mathbf{v}_{h} \in X_{h}} \frac{\left|b\left(\mathbf{v}_{I, h}, \mathbf{A}_{I, h}-\mathbf{N}_{I, h}\right)\right|}{\left\|\mathbf{v}_{h}\right\|_{H(\text { curl }, \Omega)}} .
$$

On the other hand

$$
\begin{aligned}
b\left(\mathbf{v}_{I, h}, \mathbf{A}_{I, h}-\mathbf{N}_{I, h}\right) & =\overline{F\left(\mathbf{v}_{h}\right)}-\overline{a\left(\mathbf{H}_{h}, \mathbf{v}_{h}\right)}-b\left(\mathbf{v}_{I, h}, \mathbf{N}_{I, h}\right) \\
& =\overline{a\left(\mathbf{H}-\mathbf{H}_{h}, \mathbf{v}_{h}\right)}+b\left(\mathbf{v}_{I, h}, \mathbf{A}_{I}-\mathbf{N}_{I, h}\right),
\end{aligned}
$$

then

$$
\left\|\mathbf{A}_{I, h}-\mathbf{N}_{I, h}\right\|_{L^{2}\left(\Omega^{I}\right)} \leqslant \frac{c_{1}}{\beta}\left\|\mathbf{H}-\mathbf{H}_{h}\right\|_{H(\operatorname{curl} ; \Omega)}+\frac{c_{2}}{\beta}\left\|\mathbf{A}_{I}-\mathbf{N}_{I, h}\right\|_{L^{2}\left(\Omega^{I}\right)},
$$

which yields (6.4).

To obtain (6.5) we use the inf-sup condition (6.2) that in particular gives

$$
\left\|\phi_{I, h}\right\|_{h} \leqslant \frac{1}{\gamma} \sup _{\mathbf{N}_{I, h} \in Q_{h}} \frac{\left|c_{h}\left(\mathbf{N}_{I, h}, \phi_{I, h}\right)\right|}{\left\|\mathbf{N}_{I, h}\right\|_{L^{2}\left(\Omega^{I}\right)}} .
$$

On the other hand

$$
\begin{aligned}
c_{h}\left(\mathbf{N}_{I, h}, \phi_{I, h}\right) & =\overline{G\left(\mathbf{N}_{I, h}\right)}-\overline{b\left(\mathbf{H}_{I, h}, \mathbf{N}_{I, h}\right)} \\
& =\overline{b\left(\mathbf{H}_{I}, \mathbf{N}_{I, h}\right)}+c\left(\mathbf{N}_{I, h}, \phi_{I}\right)-\overline{b\left(\mathbf{H}_{I, h}, \mathbf{N}_{I, h}\right)} \\
& =\overline{b\left(\mathbf{H}_{I}-\mathbf{H}_{I, h}, \mathbf{N}_{I, h}\right)},
\end{aligned}
$$

then

$$
\left\|\phi_{I, h}\right\|_{h} \leqslant \frac{c_{2}}{\gamma}\left\|\mathbf{H}-\mathbf{H}_{h}\right\|_{H(\operatorname{curl}, \Omega)}
$$

REMARK 6.1 It is worthy to note that $Q_{h}^{I, 0}=\epsilon^{-1} \operatorname{curl} X_{h}^{I}$ and that there exists $\mathbf{q}_{I} \in$ $H_{0, \partial \Omega}\left(\operatorname{curl} ; \Omega^{I}\right)$ such that $\boldsymbol{\epsilon} \mathbf{A}_{I}=\operatorname{curl} \mathbf{q}_{I}$. Hence

$$
\begin{aligned}
\inf _{\mathbf{N}_{I, h} \in Q_{h}^{I, 0}}\left\|\mathbf{A}_{I}-\mathbf{N}_{I, h}\right\|_{L^{2}\left(\Omega^{I}\right)} & =\inf _{\mathbf{q}_{I, h} \in X_{h}^{I}}\left\|\boldsymbol{\epsilon}^{-1}\left(\operatorname{curl} \mathbf{q}_{I}-\operatorname{curl} \mathbf{q}_{I, h}\right)\right\|_{L^{2}\left(\Omega^{I}\right)} \\
& \leqslant C \inf _{\mathbf{q}_{I, h} \in X_{h}^{I}}\left\|\mathbf{q}_{I}-\mathbf{q}_{I, h}\right\|_{H\left(\operatorname{curl} ; \Omega^{I}\right)} .
\end{aligned}
$$




\section{REFERENCES}

Alonso, A. \& VAlLi, A. (1996) Some remarks on the characterization of the space of tangential traces of $H(\operatorname{rot} ; \Omega)$ and the construction of an extension operator. Manuscripta Math., 89, 159178 .

Alonso, A. \& VAlli, A. (1999) An optimal domain decomposition preconditioner for lowfrequency time-harmonic Maxwell equations. Math. Comput., 68, 607-631.

Alonso Rodríguez, A., Fernandes, P. \& Valli, A. (2003a) The time-harmonic eddy-current problem in general domains: solvability via scalar potentials. Computational Electromagnetics, Lecture Notes in Computational Science and Engineering, Vol. 28. (C. Carstensen, S. Funken, W. Hackbusch, R. H. W. Hoppe \& P. Monk, eds). Berlin: Springer, pp. 143-163.

Alonso Rodríguez, A., Fernandes, P. \& VAlli, A. (2003b) Weak and strong formulations for the time-harmonic eddy-current problem in general domains. Eur. J. Appl. Math., 14, 387-406.

Ammari, H., Buffa, A. \& NÉDÉlEC, J.-C. (2000) A justification of Eddy currents model for the Maxwell equations. SIAM J. Appl. Math., 60, 1805-1823.

Amrouche, C., Bernardi, C., Dauge, M. \& Girault, V. (1998) Vector potentials in threedimensional nonsmooth domains. Math. Methods Appl. Sci., 21, 823-864.

Bermúdez, A., Rodríguez, R. \& SALGAdo, P. (2002) A finite element method with Lagrange multipliers for low-frequency harmonic Maxwell equations. SIAM J. Numer. Anal., 40, 18231849.

BossAVIT, A. (1985) Two dual formulations of the 3D eddy-currents problem. COMPEL, 4, 103116.

Bossavit, A. (1998) Computational Electromagnetism. San Diego, CA: Academic.

BrezZI, F. \& Fortin, M. (1991) Mixed and Hybrid Finite Element Methods. New York: Springer.

Chen, Z., DU, Q. \& Zou, J. (2000) Finite element methods with matching and non-matching meshes for Maxwell equations with discontinuous coefficients. SIAM J. Numer. Anal., 37, 15421570.

DIRKS, H. K. (1996) Quasi-stationary fields for microelectronic applications. Electr. Engng, 79, $145-155$.

FERNANDES, P. \& GilARDI, G. (1997) Magnetostatic and electrostatic problems in inhomogeneous anisotropic media with irregular boundary and mixed boundary conditions. Math. Models Meth. Appl. Sci., $M^{3} A S$, 7, 957-991.

Girault, V. \& RaViart, P. A. (1986) Finite Element Methods for Navier-Stokes Equations. Berlin: Springer.

Gross, P. W. \& KotiUgA, P. R. (2001) Finite element-based algorithms to make cuts for magnetic scalar potentials: topological constraints and computational complexity. Geometric Methods for Computational Electromagnetics, PIER, 32. (F. L. Teixeira, ed.). Cambridge: EMW Publishing, pp. 207-245.

HiptmaiR, R. (2002) Finite elements in computational electromagnetism. Acta Numerica, 237-339.

Monk, P. (1991) A mixed method for approximating Maxwell's equations. SIAM J. Numer. Anal., 28, 1610-1634.

Silvester, P. P. \& Ferrari, R. L. (1990) Finite Elements for Electrical Engineers. Cambridge: Cambridge University Press.

TARHASAARI, T. \& KetTunen, L. (2001) Topological approach to computational electromagnetism. Geometric Methods for Computational Electromagnetics, PIER, 32. (F. L. Teixeira, ed.). Cambridge: EMW Publishing, pp. 189-206. 